Supporting Information

\title{
Theoretical Study on the Light-emitting Mechanism of
}

\section{Multifunctional Thermally Activated Delayed Fluorescence}

\section{Molecules}

Kai Zhang\#, Qun Zhang\#, Muzhen Li, Yuzhi Song, Jianzhong Fan, Chuan-Kui

Wang*, Lili Lin*

Shandong Key Laboratory of Medical Physics and Image Processing \& Shandong

Provincial Engineering and Technical Center of Light Manipulations, School of

Physics and Electronics, Shandong Normal University, Jinan 250358, China

\section{Corresponding Author}

\# Authors who contribute equally to the work.

*Author to whom correspondence should be addressed.

E-mail: ckwang@sdnu.edu.cn and linll@sdnu.edu.cn 


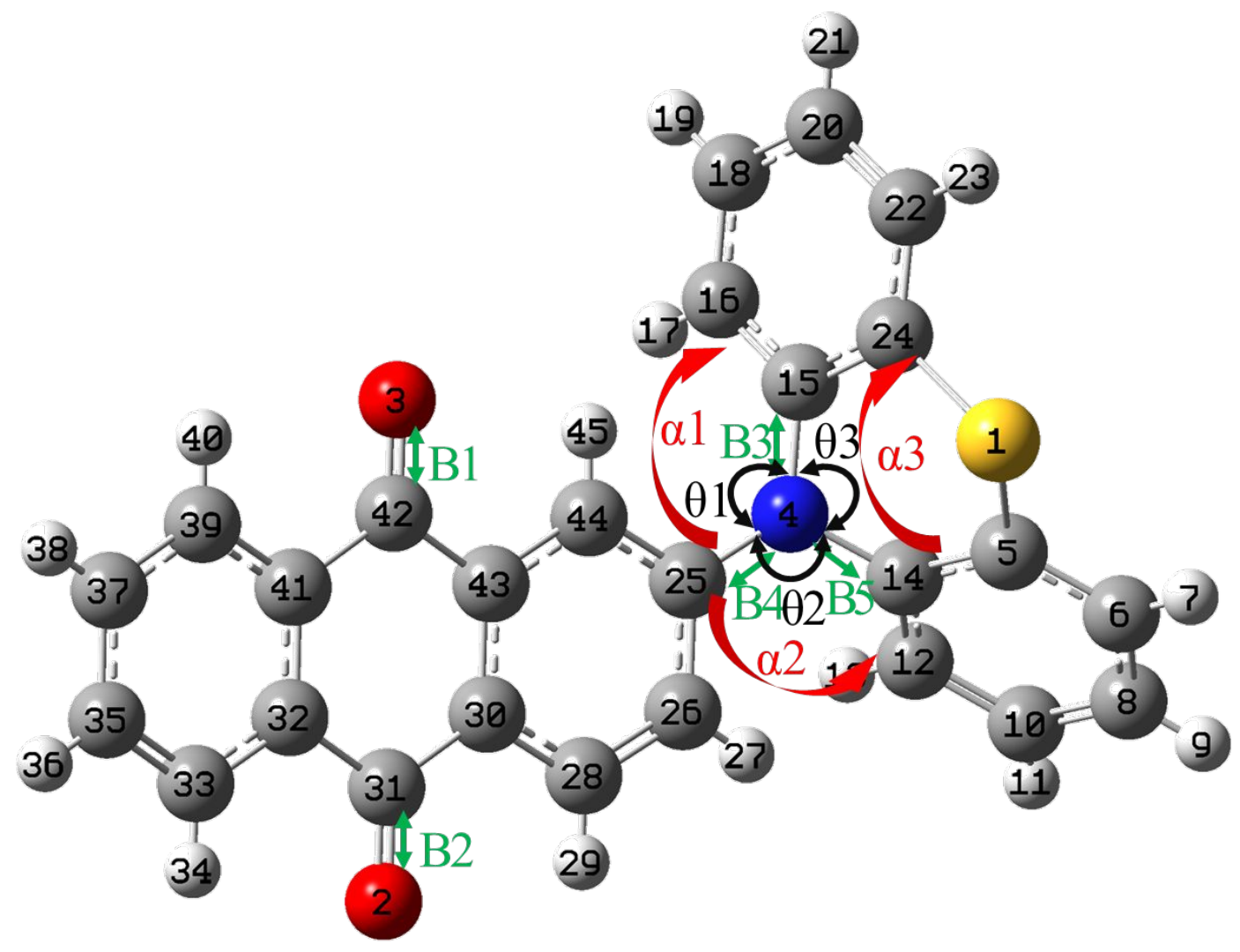

Figure S1. Atomic label, the interesting bond distances, bond angles and dihedral angles of PTZ-AQ. (B1:3-42; B2: 2-31; B3:4-15; B4:4-25; B5:4-14; $01: 15-4-25$; $\theta 2: 25-4-14 ; \theta 3: 15-4-14 ; \alpha 1: 25-4-15-16 ; \alpha 2: 25-4-14-12 ; \alpha 3: 14-5-1-24)$ 
(a)

(1)

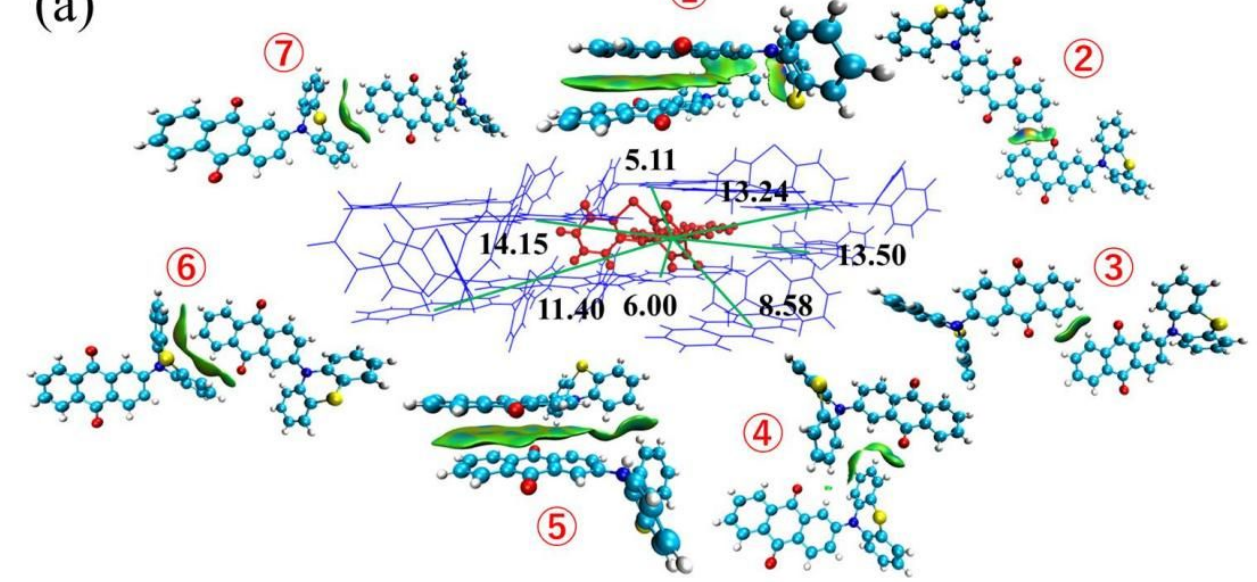

(b)

(1)

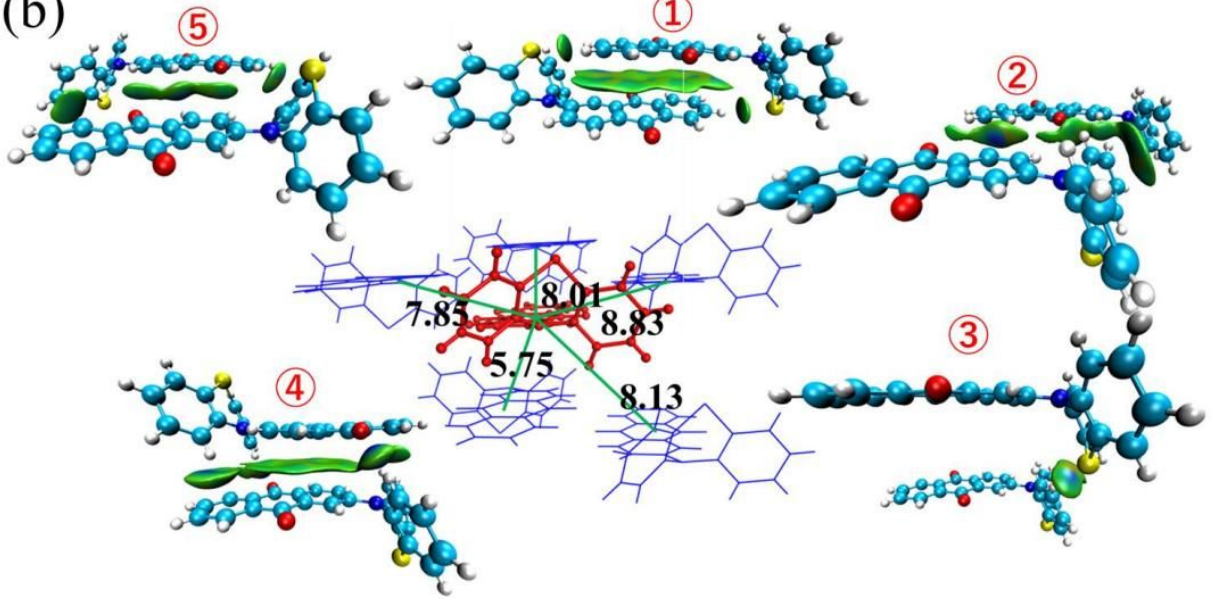

(c)
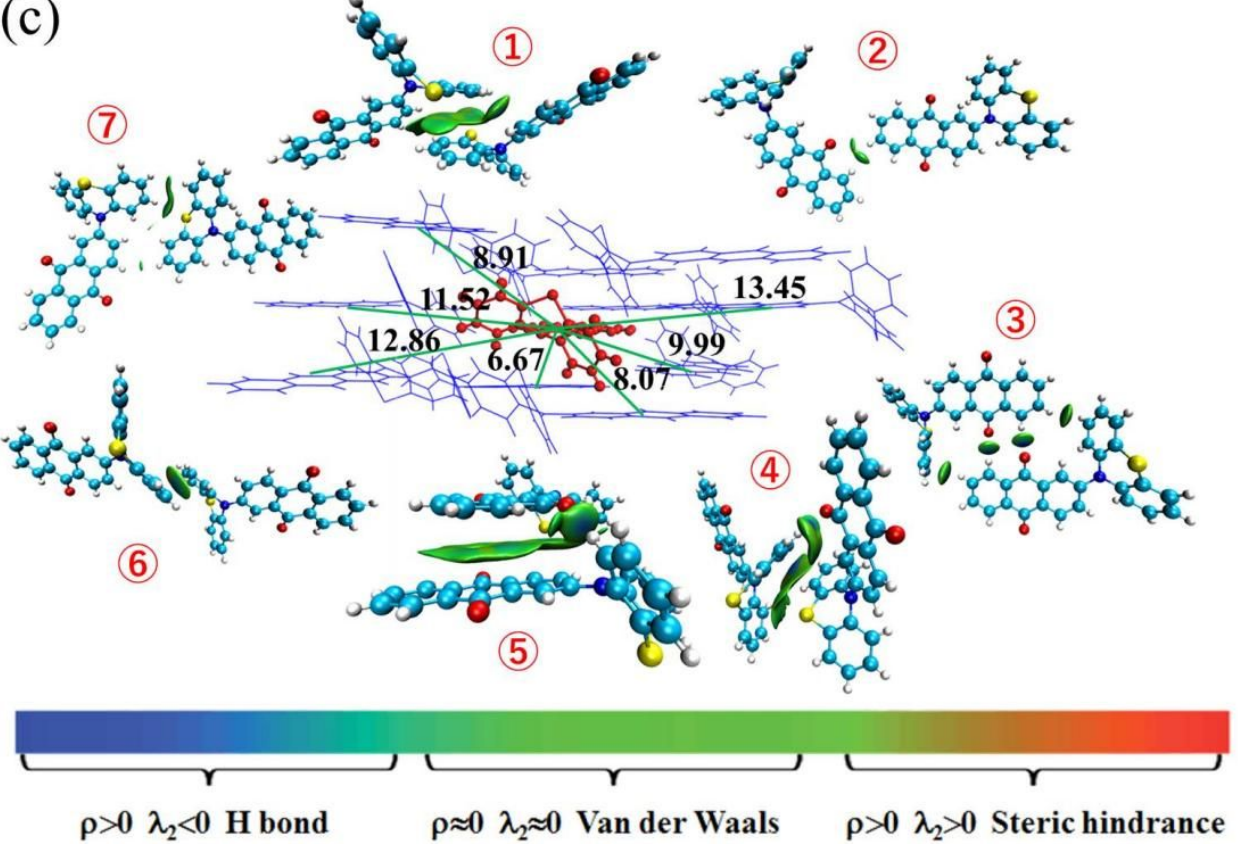

Figure S2. Intermolecular interactions for several dimers described for three aggregation states (Y-crystal (a), O-crystal (b) and R-crystal (c)) by the IGM method. 
(a)

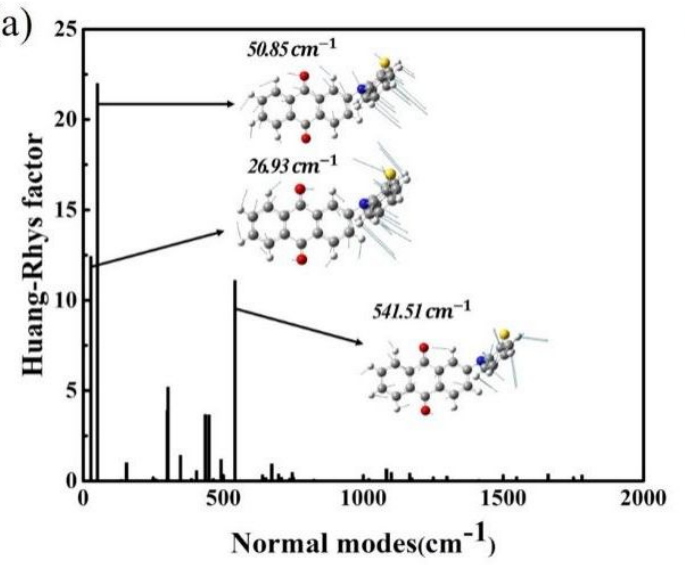

(b)

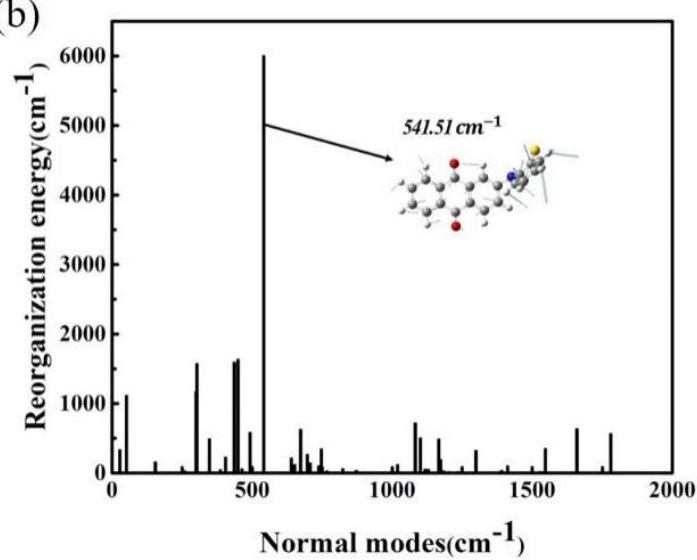

Figure S3. Calculated HR factor (a) and the reorganization energy (b) versus the normal mode frequencies for PTZ-AQ-1 for energy conversion between $S_{1}$ and $S_{0}$ respectively, as well as the representative vibration modes are depicted.

Table S1 Geometry parameters of $\mathrm{S}_{0}, \mathrm{~S}_{1}, \mathrm{~T}_{1}$ and $\mathrm{T}_{2}$ states for PTZ-AQ-Y respectively. All the parameters are shown in Figure S1.

\begin{tabular}{lccccc}
\hline & Exp. & $\mathbf{S}_{\mathbf{0}}$ & $\mathbf{S}_{\mathbf{1}}$ & $\mathbf{T}_{\mathbf{1}}$ & $\mathbf{T}_{\mathbf{2}}$ \\
\hline $\mathbf{B 1}$ & 1.20 & 1.22 & 1.25 & 1.24 & 1.29 \\
$\mathbf{B 2}$ & 1.21 & 1.22 & 1.24 & 1.25 & 1.23 \\
$\mathbf{B 3}$ & 1.41 & 1.42 & 1.40 & 1.42 & 1.42 \\
$\mathbf{B 4}$ & 1.43 & 1.40 & 1.44 & 1.39 & 1.40 \\
$\mathbf{B 5}$ & 1.44 & 1.42 & 1.40 & 1.42 & 1.42 \\
$\boldsymbol{\theta 1}$ & 120.1 & 119.6 & 118.8 & 119.9 & 119.4 \\
$\boldsymbol{\theta 2}$ & 119.0 & 123.1 & 123.4 & 124.3 & 123.0 \\
$\boldsymbol{\theta 3}$ & 117.7 & 115.4 & 116.9 & 115.3 & 115.5 \\
$\boldsymbol{\alpha 1}$ & -66.9 & -62.3 & -52.6 & -55.9 & -63.0 \\
$\boldsymbol{\alpha 2}$ & 70.5 & 66.8 & 59.0 & 60.7 & 67.7 \\
$\boldsymbol{\alpha 3}$ & -34.6 & -36.1 & -31.4 & -34.5 & -36.1 \\
\hline
\end{tabular}


Table $\mathrm{S} 2$ Geometry parameters of $\mathrm{S}_{0}, \mathrm{~S}_{1}, \mathrm{~T}_{1}$ and $\mathrm{T}_{2}$ states for PTZ-AQ-O respectively. All the parameters are shown in Figure S1.

\begin{tabular}{cccccc}
\hline & Exp. & $\mathbf{S}_{\mathbf{0}}$ & $\mathbf{S}_{\mathbf{1}}$ & $\mathbf{T}_{\mathbf{1}}$ & $\mathbf{T}_{\mathbf{2}}$ \\
\hline B1 & 1.21 & 1.22 & 1.25 & 1.25 & 1.23 \\
$\mathbf{B 2}$ & 1.22 & 1.22 & 1.24 & 1.24 & 1.30 \\
$\mathbf{B 3}$ & 1.43 & 1.42 & 1.40 & 1.40 & 1.42 \\
$\mathbf{B 4}$ & 1.41 & 1.40 & 1.45 & 1.45 & 1.40 \\
$\mathbf{B 5}$ & 1.43 & 1.42 & 1.40 & 1.40 & 1.42 \\
$\boldsymbol{\theta 1}$ & 120.1 & 120.6 & 120.7 & 120.8 & 120.5 \\
$\boldsymbol{\theta 2}$ & 120.5 & 120.8 & 121.7 & 121.6 & 121.0 \\
$\boldsymbol{\theta 3}$ & 115.3 & 115.3 & 116.5 & 116.5 & 115.3 \\
$\boldsymbol{\alpha 1}$ & -69.9 & -68.3 & -54.5 & -54.5 & -67.7 \\
$\boldsymbol{\alpha 2}$ & 69.9 & 68.8 & 57.6 & 57.6 & 68.3 \\
$\boldsymbol{\alpha 3}$ & -34.8 & -32.4 & -29.5 & -29.5 & -32.6 \\
\hline
\end{tabular}

Table S3 Geometry parameters of $\mathrm{S}_{0}, \mathrm{~S}_{1}, \mathrm{~T}_{1}$ and $\mathrm{T}_{2}$ states for PTZ-AQ-R respectively. All the parameters are shown in Figure S1.

\begin{tabular}{cccccc}
\hline & Exp. & $\mathbf{S}_{\mathbf{0}}$ & $\mathbf{S}_{\mathbf{1}}$ & $\mathbf{T}_{\mathbf{1}}$ & $\mathbf{T}_{\mathbf{2}}$ \\
\hline B1 & 1.22 & 1.22 & 1.25 & 1.25 & 1.23 \\
$\mathbf{B 2}$ & 1.22 & 1.22 & 1.24 & 1.25 & 1.29 \\
$\mathbf{B 3}$ & 1.44 & 1.42 & 1.40 & 1.42 & 1.42 \\
$\mathbf{B 4}$ & 1.40 & 1.40 & 1.46 & 1.40 & 1.40 \\
$\mathbf{B 5}$ & 1.43 & 1.42 & 1.40 & 1.42 & 1.42 \\
$\boldsymbol{\theta 1}$ & 120.0 & 121.3 & 119.5 & 120.9 & 121.0 \\
$\boldsymbol{\theta 2}$ & 123.0 & 123.2 & 123.3 & 123.9 & 123.3 \\
$\boldsymbol{\theta 3}$ & 115.1 & 115.1 & 116.6 & 115.0 & 115.1 \\
$\boldsymbol{\alpha 1}$ & -63.5 & -58.6 & -51.4 & -53.6 & -58.6 \\
$\boldsymbol{\alpha 2}$ & 65.8 & 59.8 & 55.2 & 55.5 & 59.9 \\
$\boldsymbol{\alpha 3}$ & -39.0 & -38.9 & -34.6 & -36.7 & -38.9 \\
\hline
\end{tabular}


Table S4 Intermolecular interaction energy including electronic, repulsion, and dispersion interactions in several dimers extracted from the three crystal structures.

\begin{tabular}{|c|c|c|c|c|c|c|}
\hline & Dimer & $\begin{array}{l}\text { Centroid } \\
\text { distance }\end{array}$ & Electrostatic & Repulsion & Dispersion & Total \\
\hline \multirow{7}{*}{$\begin{array}{l}\text { Y-crys } \\
\text { tal }\end{array}$} & dimer-1 & 5.11 & 5.36 & 46.04 & -117.05 & -65.65 \\
\hline & dimer-2 & 13.24 & -4.37 & 2.47 & -10.57 & -12.48 \\
\hline & dimer-3 & 13.50 & -2.84 & 1.37 & -8.06 & -9.53 \\
\hline & dimer-4 & 8.58 & -6.52 & 4.31 & -21.08 & -23.30 \\
\hline & dimer-5 & 6.00 & 4.89 & 39.78 & -98.60 & -53.92 \\
\hline & dimer-6 & 11.40 & -3.91 & 7.02 & -26.81 & -23.70 \\
\hline & dimer-7 & 14.15 & -1.33 & 2.34 & -11.67 & -10.67 \\
\hline \multirow{5}{*}{$\begin{array}{l}\text { O-crys } \\
\text { tal }\end{array}$} & dimer-1 & 8.01 & 0.69 & 34.63 & -92.63 & -57.32 \\
\hline & dimer-2 & 8.83 & -8.27 & 8.09 & -28.71 & -28.90 \\
\hline & dimer-3 & 8.13 & -0.52 & 3.16 & -14.57 & -11.93 \\
\hline & dimer-4 & 5.75 & -13.68 & 28.83 & -97.23 & -82.08 \\
\hline & dimer-5 & 7.85 & 1.61 & 8.97 & -35.39 & -25.79 \\
\hline \multirow{7}{*}{$\begin{array}{l}\text { R-crys } \\
\text { tal }\end{array}$} & dimer-1 & 8.91 & -2.88 & 18.97 & -52.19 & -36.09 \\
\hline & dimer-2 & 13.45 & -3.04 & 0.66 & -5.61 & -7.99 \\
\hline & dimer-3 & 9.99 & -0.93 & 6.22 & -23.39 & -18.10 \\
\hline & dimer-4 & 8.07 & -2.09 & 17.16 & -50.15 & -35.08 \\
\hline & dimer-5 & 6.67 & -2.66 & 19.65 & -66.77 & -49.78 \\
\hline & dimer-6 & 12.86 & -1.43 & 3.25 & -10.29 & -8.47 \\
\hline & dimer-7 & 11.52 & -0.18 & 1.87 & -12.69 & -10.99 \\
\hline
\end{tabular}


Table S5 The relative energy and Boltzmann ratio of the $T_{1}, T_{2}$ and $T_{3}$ states.

\begin{tabular}{|c|c|c|}
\hline Conformation & $\Delta E(m e V)$ & Proportion \\
\hline PTZ-AQ-Y -T 1 & 0 & $57 \%$ \\
\hline PTZ-AQ-Y -T & 480 & $25 \%$ \\
\hline PTZ-AQ-Y -T 3 & 670 & $18 \%$ \\
\hline PTZ-AQ-O -T 1 & 0 & $57 \%$ \\
\hline PTZ-AQ-O -T 2 & 490 & $25 \%$ \\
\hline PTZ-AQ-O - $\mathbf{T}_{3}$ & 690 & $18 \%$ \\
\hline PTZ-AQ-R -T 1 & 0 & $55 \%$ \\
\hline PTZ-AQ-R -T 2 & 450 & $26 \%$ \\
\hline PTZ-AQ-R - $\mathbf{T}_{3}$ & 650 & $19 \%$ \\
\hline
\end{tabular}

Table S6 Twist angles and relative energies of $\mathrm{S}_{0}$ and $\mathrm{S}_{1}$ states for PTZ-AQ shown in Figure S1 respectively.

\begin{tabular}{|c|c|c|c|}
\hline \multicolumn{2}{|c|}{$\overline{S_{0}}$} & \multicolumn{2}{|c|}{$\overline{S_{1}}$} \\
\hline Angle ( $\left(^{\circ}\right)$ & $\operatorname{Energy}(e V)$ & Angle $\left(^{\circ}\right)$ & $\operatorname{Energy}(e V)$ \\
\hline-173 & 0 & -90 & 1.522 \\
\hline-120 & 0.201 & -17 & 2.372 \\
\hline-100 & 0.112 & 90 & 1.52 \\
\hline-48 & 0.201 & 153 & 2.372 \\
\hline 7 & 0 & ---- & ---- \\
\hline 60 & 0.188 & ---- & ---- \\
\hline 80 & 0.109 & ---- & ---- \\
\hline 130 & 0.188 & ---- & ---- \\
\hline
\end{tabular}


Table S7 Twist angles and relative energies of $\mathrm{S}_{0}$ and $\mathrm{S}_{1}$ states, the emission wavelengths and oscillator strengths for Y-aggregation.

\begin{tabular}{cc|ccc}
\hline & $\mathbf{S}_{\mathbf{0}}$ & & & \multicolumn{3}{|c}{$\mathbf{S}_{\mathbf{1}}$} & \\
Angle $\left(^{\circ}\right)$ & Energy(eV) & Energy (eV) & Wave(nm) & $\boldsymbol{f}$ \\
\hline-41 & 0.211 & 2.546 & 616 & 0.0905 \\
-36 & 0.162 & 2.547 & 602 & 0.0925 \\
-31 & 0.116 & 2.552 & 588 & 0.0940 \\
-26 & 0.078 & 2.576 & 568 & 0.0976 \\
-21 & 0.048 & 2.565 & 562 & 0.0950 \\
-16 & 0.025 & 2.561 & 557 & 0.0921 \\
-11 & 0.010 & 2.562 & 553 & 0.0888 \\
-6 & 0.002 & 2.572 & 548 & 0.0857 \\
-1 & 0 & 2.616 & 543 & 0.0825 \\
4 & 0.003 & 2.638 & 538 & 0.0793 \\
9 & 0.009 & 2.668 & 534 & 0.0748 \\
14 & 0.019 & 2.708 & 531 & 0.0710 \\
19 & 0.039 & 2.757 & 528 & 0.0676 \\
24 & 0.069 & 2.546 & 525 & 0.0643 \\
\hline
\end{tabular}

Table S8 Twist angles and relative energies of $\mathrm{S}_{0}$ and $\mathrm{S}_{1}$ states, the emission wavelengths and oscillator strengths for O-aggregation.

\begin{tabular}{cc|ccc}
\hline & & $\mathbf{S}_{\mathbf{0}}$ & & \multicolumn{3}{|c}{$\mathbf{S}_{\mathbf{1}}$} \\
Angle $\left(^{\circ}\right)$ & Energy(eV) & Energy $(\mathbf{e V})$ & Wave(nm) & $\boldsymbol{f}$ \\
\hline-24 & 0.127 & 2.640 & 570 & 0.0926 \\
-19 & 0.118 & 2.602 & 568 & 0.0889 \\
-14 & 0.080 & 2.574 & 566 & 0.0851 \\
-9 & 0.050 & 2.556 & 564 & 0.0815 \\
-4 & 0.026 & 2.547 & 562 & 0.0779 \\
1 & 0.010 & 2.546 & 559 & 0.0744 \\
6 & 0 & 2.553 & 557 & 0.0711 \\
11 & 0.002 & 2.569 & 554 & 0.0678 \\
16 & 0.019 & 2.593 & 552 & 0.0646 \\
21 & 0.045 & 2.626 & 549 & 0.0613 \\
26 & 0.082 & 2.665 & 547 & 0.0581 \\
31 & 0.129 & 2.713 & 546 & 0.0549 \\
36 & 0.147 & 2.767 & 542 & 0.0515 \\
\hline
\end{tabular}


Table S9 Twist angles and relative energies of $\mathrm{S}_{0}$ and $\mathrm{S}_{1}$ states, the emission wavelengths and oscillator strengths for R-crystal.

\begin{tabular}{cc|ccc}
\hline \multicolumn{2}{c|}{$\mathbf{S}_{\mathbf{0}}$} & & \multicolumn{3}{|c}{$\mathbf{S}_{\mathbf{1}}$} \\
Angle $\left(^{\circ}\right)$ & Energy(eV) & Energy $(\mathbf{e V})$ & Wave(nm) & $\boldsymbol{f}$ \\
\hline-42 & 0.199 & 2.505 & 627 & 0.0949 \\
-37 & 0.142 & 2.467 & 621 & 0.0938 \\
-32 & 0.095 & 2.436 & 615 & 0.0921 \\
-27 & 0.059 & 2.413 & 610 & 0.0898 \\
-22 & 0.031 & 2.398 & 606 & 0.0871 \\
-17 & 0.013 & 2.392 & 602 & 0.0842 \\
-12 & 0.002 & 2.395 & 598 & 0.0812 \\
-7 & 0 & 2.408 & 594 & 0.0780 \\
-2 & 0.006 & 2.430 & 590 & 0.0749 \\
37 & 0.020 & 2.461 & 586 & 0.0718 \\
8 & 0.042 & 2.503 & 581 & 0.0688 \\
13 & 0.074 & 2.554 & 577 & 0.0659 \\
18 & 0.114 & 2.614 & 573 & 0.0630 \\
\hline
\end{tabular}

